\title{
Eine seltene Genodermatose: Erythrokeratodermia variabilis et figurata Mendes da Costa
}

\author{
A Rare Genodermatosis: Erythrokeratodermia Variabilis et \\ Figurata Mendes da Costa
}

Autoren

Institut

\section{H. Khan Durani, W. Hartschuh}

Universitäts-Hautklinik Heidelberg

\section{Bibliografie}

DOI 10.1055/s-2007-995599

Akt Dermatol 2008; 34 :

90-92 ๑ Georg Thieme

Verlag KG Stuttgart · New York ISSN 0340-2541

Korrespondenzadresse Dr. med. Hendrike Khan Durani

Universitäts-Hautklinik Heidelberg

Voßstr. 2

69115 Heidelberg Hendrike_Durani@med.uniheidelberg.de

\section{Zusammenfassung \\ $\nabla$}

Die Erythrokeratodermia variabilis et figurata Mendes da Costa gehört zu der Gruppe der Genodermatosen. Diese beginnt meist in frühester Kindheit und ist von stationären Hyperkeratosen und wandernden Erythemen gekennzeichnet.

\section{Einleitung}

Die Erythrokeratodermia variabilis et figurata ist eine seltene, autosomal dominant vererbte Genodermatose, die wandernde Erytheme und stationäre Hyperkeratosen aufweist. Die Ausprägung der Morphen ist individuell, intra- und interfamiliär stark variabel. Das Manifestationsalter liegt in der Regel im ersten Lebensjahr. 1998 wurde erstmalig ein zugrunde liegender Gendefekt beschrieben [1].

\section{Fallbericht \\ $\nabla$}

\section{Anamnese}

Bei der jetzt 55-jährigen Patientin treten seit frühester Kindheit großflächige rötliche, wandernde Erytheme sowie Hyperkeratosen auf. Die Erytheme zeigen sich variabel in der Konfiguration und der Ausdehnung am gesamten Integument mit Aussparung des Gesichtes. Sie blassen innerhalb von Stunden bis Tagen ab und treten manchmal mit einer brennenden Sensation auf. Zusätzlich zeigen sich seit Jahren persistierende, hyperkeratotische bräunliche Plaques, v.a. im Bereich des Abdomen, sacral, der Mammae und an den oberen und unteren Extremitäten. Palmae und Plantae sind frei. Eine Zunahme der Hyperkeratosen besteht seit dem 45. Lebensjahr. In den Sommermonaten sowie in stressigen Situationen berichtet die Patientin über verstärkte Erythembildung.
$50 \%$ der Patienten weisen auch palmoplantare Hyperkeratosen auf. Der Gendefekt liegt auf dem Chromosom 1p34-35.1. Die Therapie erfolgt mit keratolytischen und pflegenden Lokaltherapien, bei schwereren Verlaufsformen systemisch mit Acitretin.

Die Schwester, der Bruder sowie der Vater leiden an derselben Hautkrankheit. Über die Großeltern konnte keine Angabe gemacht werden, da diese früh verstorben sind. Weitere Nachfahren gibt es nicht.

\section{Dermatologischer Aufnahmebefund}

Bräunliche Hyperkeratosen befinden sich verstärkt im Bereich des Abdomen, sakral, um die Axillae und Mammae. V.a. im Bereich dieser Hyperkeratosen zeigen sich girlandenartige Erytheme mit scharfer Begrenzung ( $\bullet$ Abb. 1, 2).

\section{Histologie}

Unregelmäßige Akanthose mit Papillomatose sowie Orthohyperkeratose bei diskreter, kutan-vaskulärer Entzündung des oberen Coriums.

Eine Immunhistologie oder Elektronenmikroskopie wurde nicht durchgeführt.

\section{Labor}

Es zeigten sich bis auf leicht erhöhte Werte für die Harnsäure und Leukozyten keinerlei Auffälligkeiten im Routinelabor.

\section{Therapie und Verlauf $\nabla$}

Diverse keratolytische und rückfettende Lokaltherapien erbrachten nicht den gewünschten Erfolg, sodass wir mit einer Acitretin-Therapie $0,5 \mathrm{mg} / \mathrm{kg}$ KG begannen. Hierunter zeigte sich 


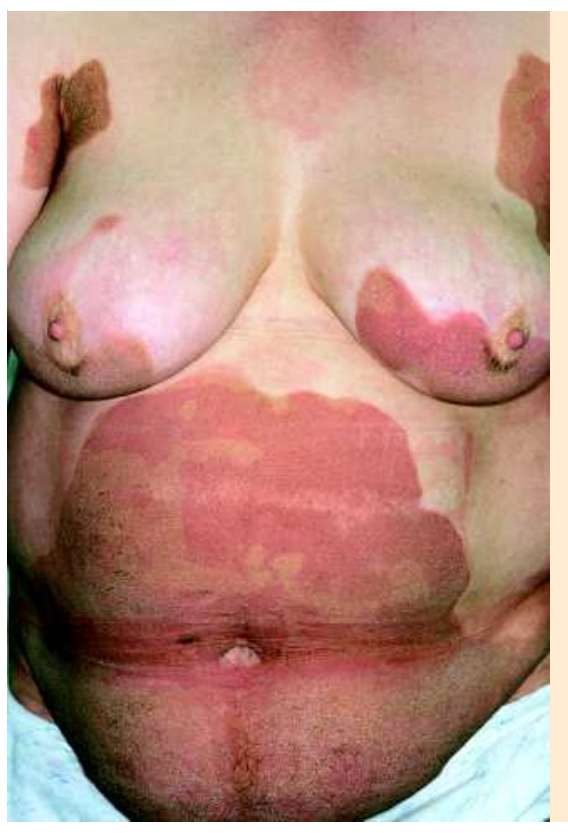

Abb. 1 Hyperkeratosen und Erytheme.

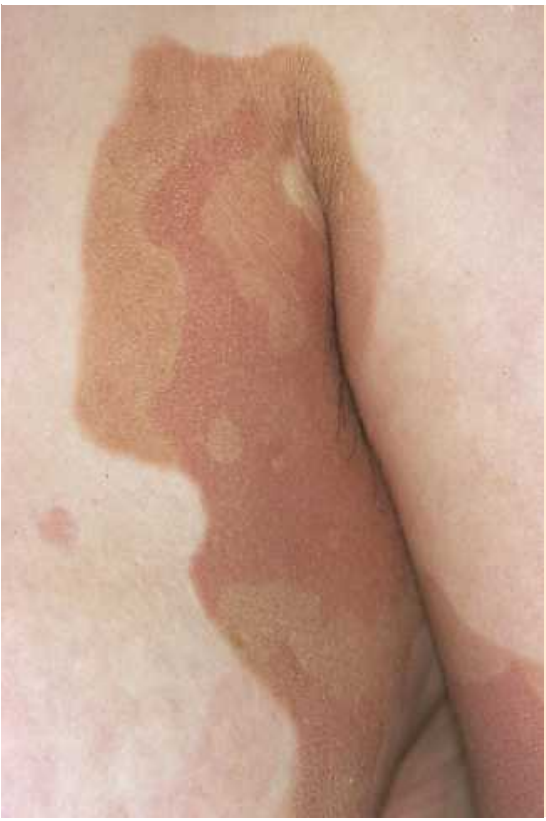

Abb. 2 Detailaufnahme Oberkörper/Arm.

innerhalb weniger Wochen ein Rückgang der Keratosen, sodass wir nach kurzer Zeit die Acitretin-Dosis auf 0,25 mg/kg Körpergewicht reduzieren konnten $(\bullet$ Abb. 3).

\section{Diskussion \\ $\nabla$}

Mendes da Costa berichtete 1925 detailliert von der Erythrokeratodermia variabilis [2]. Es handelt sich um eine seltene aut.dom. mit beinahe kompletter Penetranz vererbten Genodermatose mit epidermaler Verhornungsstörung. Typisch hierfür sind einerseits wandernde Erytheme, andererseits die persistierenden bräunlichen Hyperkeratosen. Die Hauteffloreszenzen heilen in der Regel residuenlos ab. 50\% der Patienten weisen zusätzlich eine palmoplantare Hyperkeratose auf. Die Ausprägung des Hautbildes ist individuell und interfamiliär stark variabel. Die Erythembildung zeigt sich meist vermehrt in der Kindheit, bei

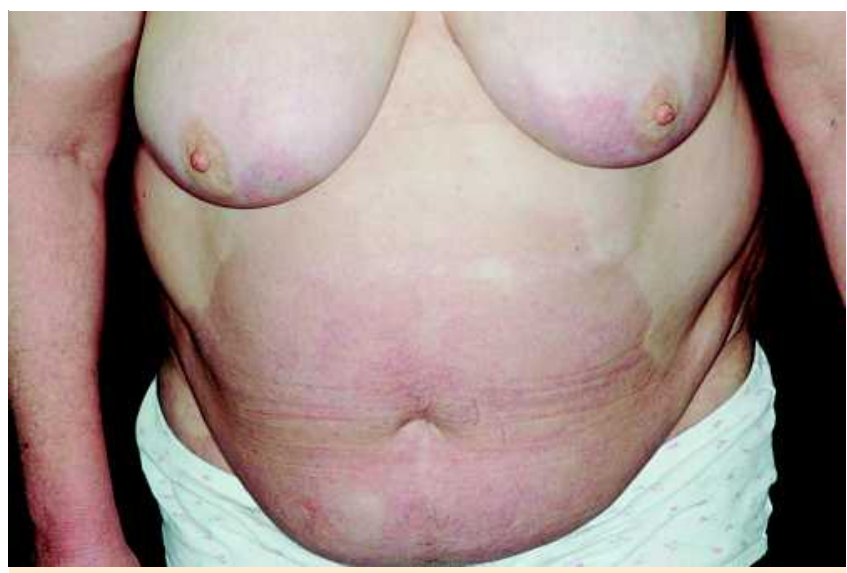

Abb. 3 Nach 6 wöchiger Neotigason-Therapie.

manchen Patienten stabilisiert sich die Erkrankung nach der Pubertät [3]. Zum Teil wird über brennende Hyperästhesien im Bereich der Erytheme berichtet. Als Triggerfaktoren werden physikalische Faktoren (Kälte/Wärme), Stress, hormonelle Umstellungen sowie mechanische Irritationen angegeben.

1998 wurde erstmalig der Gendefekt für die Erkrankung beschrieben [3]. Seitdem wurden weitere Loci beschrieben, alle liegen auf dem Chromosom 1p34-35.1. Der kurze Arm von Chromosom 1 ist für die Kodierung der Connexin Gene GJB3 und GJB4 verantwortlich. Diese Gene kodieren die Gap junction Proteine Connexin 31 und Connexin 30.3. Sie gehören zu einer großen Gruppe von transmembranösen Proteinen (Connexinen), die interzelluläre Kanäle formieren, welche wiederum für die Signalweiterleitung (oder besser: den Austausch von Botenstoffen und Metaboliten) zwischen benachbarten Zellen wichtig sind. Diese Kommunikation spielt u.a. eine wichtig Rolle bei der Zellproliferation und -differenzierung in der Epidermis. Sechs Connexine bilden ein Connexon (Halbkanal), zwei aneinander gelagerte Connexone von benachbarten Zellen einen durchgehenden Kanal. Gap junction Plaques bestehen aus einer Vielzahl dieser Kanäle.

Die Mutation von Connexin 31 und 30.3 wird als ursächlich für die Erythrokeratodermia angesehen. Allerdings hat es sich gezeigt, dass nicht alle Patienten diese Form der Mutation aufweisen [4].

Die histologischen Veränderungen sind unspezifisch (Akanthose und Hyperkeratose, mildes perivaskuläres lymphozytisches Infiltrat in der Dermis). Elektronenmikroskopische Untersuchungen können eine verminderte Anzahl an Odland bodies (Keratinosomen) im Stratum granulosum zeigen mit einer großen Anzahl an verklumpten perinukleären Tonofillamenten im Stratum spinosum [5].

Die Behandlung der Erythrokeratodermien erfolgt mit keratolytischen und rückfettenden Lokaltherapien. Schwerere Formen können mit systemischen Retinoiden therapiert werden. Nach Absetzen kommt es sehr rasch zum Wiederauftreten der Hautveränderung [6]. Einmalig wurde in der Literatur über einen Fall berichtet, bei dem systemische Retinoide sich nicht wirksam zeigten [7].

Differenzialdiagnostisch muss eine progressive symmetrische Erythrokeratodermia Darier-Gottron ausgeschlossen werden. Hier imponieren an der Haut symmetrische flächenhafte scharf begrenzte Erytheme mit Hyperkeratosen v.a. an den Extremitäten. Die Hautveränderungen sind stationär. Palmoplantare Be- 
teiligungen werden hier öfter gesehen als bei der Erythrokeratodermia variabilis.

\section{Abstract}

\section{A Rare Genodermatosis: Erythrokeratodermia Variabilis et Figurata Mendes da Costa}

Erythrokeratodermia variabilis et figurata is a rare genodermatosis inherited in an autosomal dominant fashion with nearly complete penetrance, but considerable individual, intra- and interfamilial variability. The hallmark of this disease is the coexistence of stable hyperkeratosis and transient erythematous patches. They usually manifest within the first year of life. The gene defect for this disease was mentioned first in 1998.

\section{Literatur}

1 Ständer S, Stadelmann A, Traub O, Traupe H, Metze D. Erythrokeratodermia variabilis - eine Störung der epidermalen Expression von GapJunction-Proteinen. JDDG 2005; 3: 354-358

2 Mendes da Costa S. Erythro- et keratodermia variabilis in a mother and daughter. Acta Derm Venereol (stockh) 1925; 6: 255-261

3 Richard G, Smith LE, Bailey RA. Mutations in the human connexin gene GJB3 cause erythrokeratodermia variabilis. Nat Genet 1998; 68: 165 168

4 Ishida-Yamamoto AI, Kelsell D, Common J. A case of erythrokeratodermia variabilis without mutations in connexion 31. $\mathrm{Br} \mathrm{J}$ Dermatol 2000; 143: $1283-1287$

5 Rappaport IP, Goldes JA, Goltz RW. Erythrokeratodermia variabilis treated with isotretinoin. Arch Dermatol 1986; 122: 441 - 445

6 Kerkhoff van de PCM, Steijlen PM, Happle R. Acitretin in the treatment of erythrokeratodermia variabilis. Dermatologica 1990; 181: $330-$ 333

7 Erbagci Z, Tuncel AA, Denuz H. Erythrokeratodermia variabilis with adult onset: report of a sporadic case unresponsive to systemic retinoids. J Dermatol Treat 2006; 17: 187-189

\section{Buchbesprechung}

\section{Dermatologische Therapie}

C. Oster-Schmidt, T. Dirschka (Hrsg)

München: Urban \& Fischer bei Elsevier, 2. Aufl. 2007.

271 S., kart., $14,95 €$

ISBN 978-3437227301

Dieser Leitfaden passt wirklich in JEDE Kitteltasche, und ist sowohl für den Dermatologen als auch für Kollegen aus anderen Fachgebieten oft ein guter Ratgeber bei der täglichen Arbeit mit praxisbezogenen Tipps für die Patienten.

Dieses Buch bietet Ihnen eine sehr kompakte Darstellung der aktuellen dermatologischen Therapie mit Warnungen vor häufigen Behandlungsfehlern. In kurzen Steckbriefen werden die wichtigsten dermatologischen Erkrankungen erläutert, wobei Klinik und Therapie in vielen übersichtlichen Tabellen zusammengefasst werden. Des Weiteren gibt es Kapitel zu allgemeinen Therapieverfahren und Notfallmaßnahmen Damit ist der Leitfaden gerade auch bei nicht alltäglichen, aber halbwegs häufigen Hauterkrankungen gut zum Nachschlagen geeignet.

F. Oberthür, Wiesbaden 